JOURNAL OF

SYMPLECTIC GEOMETRY

Volume 6, Number 3, 247-266, 2008

\title{
HYPERSURFACES SYMPLECTIQUES RÉELLES ET PINCEAUX DE LEFSCHETZ RÉELS
}

\author{
DAMiEn GaYeT
}

In a compact symplectic real manifold, i.e., supporting an antisymplectic involution, we use Donaldson's construction to build a codimension 2 symplectic submanifold invariant under the action of the involution. If the real part of the manifold is not empty, and if the symplectic form $\omega$ is entire, then there is an integer $N$ such that for all $k$ big enough, we can find a hypersurface Poincaré dual of $N k[\omega]$ such that its real part has at least $k^{n / 2}$ connected components, up to a constant independent of $k$, and where $2 n$ is the dimension of the ambient manifold. Finally we extend to our real case Donaldson's construction of Lefschetz pencils.

Dans le cadre d'une variété symplectique compacte $X^{2 n}$ réelle, c'està-dire possédant une involution antisymplectique, nous utilisons la construction de Donaldson pour établir l'existence de sous-variétés symplectiques de codimension 2 invariantes par l'involution. Si la partie réelle de la variété est non vide, et si la forme symplectique $\omega$ est entière, alors il existe un entier $N$ tel que pour tout degré $k$ assez grand, il existe une hypersurface Poincaré duale à $N k[\omega]$, telle que sa partie réelle possède au moins $k^{n / 2}$ composantes connexes, à une constante indépendante de $k$ près, et où $2 n$ est la dimension de la variété ambiante. Enfin nous étendons au cas réel les résultats de Donaldson sur l'existence de pinceaux de Lefschetz.

\section{Introduction}

Soit $\left(X^{2 n}, \omega\right)$ une variété symplectique compacte, et supposons qu'il existe un fibré en droites complexes $L$ de classe de Chern $[\omega]$, ce qui revient à dire que les périodes de $[\omega]$ sont entières. Fixons de plus une structure presque complexe $J$ compatible avec $\omega$. Dans [1], S.K Donaldson montre qu'il existe une suite de sections $\left(s_{k}\right)_{k}$ de $L^{k}$ approximativement J-holomorphe (en abrégé $\mathrm{AH}$ ), c'est-à-dire dont le $\bar{\partial}$ est majorée par une constante indépendante de $k$, et dont la dérivée covariante est minorée aux 
endroits où $s_{k}$ s'annule par $\eta \sqrt{k}$, où $\eta$ est une constante indépendante de $k$ et strictement positive. Ainsi, pour $k$ assez grand, le lieu des zéros de $s_{k}$ est une variété lisse de codimension réelle 2. De plus, cette sous-variété tend à devenir de plus en plus $J$-complexe, si bien qu'elle devient symplectique pour $k$ assez grand.

On suppose dans cet article que $(X, \omega)$ possède une structure réelle, c'està-dire une involution $c$ vérifiant $c^{*} \omega=-\omega$. Peut-on alors constuire une suite de sections $\left(s_{k}\right)_{k}$ de $L^{k}$ du type Donaldson, et dont le lieu d'annulation est invariant par $c$ ? La réponse est positive, et tient dans le

Théorème 1.1. Soit $(X, \omega, c)$ une variété symplectique réelle. Alors il existe une hypersurface symplectique invariante par c. Plus précisément, il existe une forme symplectique $\tilde{\omega}$ aussi proche de $\omega$ qu'on veut, et un entier $N$ tel que pour tout $k$ assez grand, il existe une hypersurface symplectique réelle Poincaré duale à $N k[\tilde{\omega}]$.

Topologie de la partie réelle des hypersurfaces. Un problème en géométrie réelle est de connaitre la topologie de la partie réelle, c'est-àdire l'ensemble des points invariants par $c$, des objets réels construits. Nous obtenons le résultat suivant:

Théorème 1.2. Il existe une hypersurface symplectique réelle sans partie réelle. Si la partie réelle de $X$ est non vide, il existe une forme symplectique $\tilde{\omega}$ aussi proche de $\omega$ qu'on veut, un réel $\epsilon>0$ et un entier $N$ tels que pour tout $k$ assez grand, il existe une hypersurface symplectique réelle Poincaré duale $\grave{a}[N k \tilde{\omega}]$ dont dont le nombre de composantes connexes est au moins $\epsilon k^{n / 2}$. Cette borne est à une constante près optimale, dans le sens où pour toute suite de sections $\left(s_{k}\right)_{k}$ AH et uniformément $\eta$-transverse, il existe une constante $C$ telle que le nombre de composantes connexes de la partie réelle de $s_{k}^{-1}(0)$ est inférieur à $C k^{n / 2}$.

Cas intégrable. Dans le cas où $J$ est intégrable, nous avons la proposition suivante:

Proposition 1.3. Soit $(X, \omega, J, c)$ une variété projective réelle. Alors toutes les hypersurfaces des théorèmes précédents peuvent être construites complexes.

Pinceaux de Lefschetz réels. Rappelons que si la dimension de $X$ est $2 n$, un système de coordonnées complexes $\left(z_{1}, \ldots, z_{n}\right)$ centrées en un point $x$ est dit adapté si la forme symplectique $\omega$ est $(1,1)$ et strictement positive au point $x$ pour la structure complexe induite par ces coordonnées.

Définition 1.4. Un pinceau de Lefschetz symplectique associé à une variété symplectique $(X, \omega)$ est la donnée de:

(i) Une sous-variété symplectique $N$ de codimension réelle 4.

(ii) Une application surjective $F: X-N \rightarrow \mathbb{P}^{1}$. 
(iii) Un nombre fini de points $\Delta \subset M-N$ en dehors desquels $F$ est une submersion.

De plus, ces données vérifient les modèles locaux suivants:

(iv) Pour tout point $p \in N$, il existe une carte adaptée $\left(z_{1}, \ldots, z_{n}\right)$ pour laquelle la sous-variété $N$ a pour équation locale $\left\{z_{1}=z_{2}=0\right\}$ et telle que $F=z_{2} / z_{1}$.

(v) Pour tout point $p \in \Delta$, il existe une carte adaptée $\left(z_{1}, \ldots, z_{n}\right)$ dans laquelle $F$ s'écrit $F(z)=z_{1}^{2}+\cdots+z_{n}^{2}+c$.

Enfin, si $(X, \omega)$ est munie d'une structure réelle $c$, on dira que le pinceau est réel si

$$
\bar{F}=F \circ c .
$$

Le théorème principal de [2] est le suivant:

Théorème 1.5. [2]. Soit $(X, \omega)$ une variété symplectique compacte telle que la classe de cohomologie $[\omega]$ soit entière. Alors pour $k$ assez grand, il existe un pinceau de Lefschetz dont les fibres sont Poincaré duales de $k[\omega]$.

Nous démontrons dans la deuxième partie de cet article que ce théorème s'adapte dans le cas réel:

Théorème 1.6. Soit $(X, \omega, c)$ une variété symplectique réelle, telle que $\omega$ soit entière. Alors il existe un pinceau de Lefschetz réel.

Remerciements. Je remercie I'Agence nationale de la recherche (France) pour son soutien ainsi que Jean-Yves Welschinger pour m'avoir posé cette question et pour ses judicieux commentaires tout au long de ce travail, et enfin le referee pour ses suggestions et corrections.

\section{Construction d'une hypersurface symplectique réelle}

2.1. Structures associées à la structure réelle. Structures presque complexes. Une première remarque démontrée dans [3] est qu'il existe une structure presque complexe $J$ à la fois compatible avec $\omega$ et rendant $c$ antiholomorphe, c'est-à-dire telle que $d c \circ J=-J d c$. Dans toute la suite, $J$ désignera une telle structure.

Forme symplectiques à valeurs entières. Pour que $-i \omega$ soit la courbure d'un fibré en droites complexes, il faut que ses valeurs sur les 2-cycles soient entières. Le lemme suivant nous permet de réaliser cette condition, tout en restant dans un cadre réel.

Lemme 2.1. Si $\omega$ n'est pas à valeurs rationnelles, on peut la perturber en $\tilde{\omega}$ de sorte que c reste antisymplectique pour la nouvelle forme.

Démonstration. En effet, soit $\mathcal{Z}_{+}^{2}$ le sous-espace vectoriel propre pour la valeur propre +1 de l'endomrophisme $-c^{*}$ :

$$
-c^{*}: \mathcal{Z}^{2}(X, \mathbb{R}) \rightarrow \mathcal{Z}^{2}(X, \mathbb{R})
$$


agissant sur les 2 -formes fermées réelles. Le réseau $\mathcal{Z}^{2}(X, \mathbb{Q})$ des 2 -formes fermées à valeurs rationnelles sur les 2-cycles est invariant par $-c^{*}$, et son intersection $\mathcal{Z}_{+}^{2} \cap \mathcal{Z}^{2}(X, \mathbb{Q})$ est dense dans $\mathcal{Z}_{+}^{2}$. On peut donc perturber $\omega$ en un élément de $\mathcal{Z}^{2}(X, \mathbb{Q})$ tout en restant dans l'espace des formes invariantes par $-c^{*}$.

Fibrés $c$-réels. Le lemme suivant est immédiat:

Lemme 2.2. Soit $E$ un fibré complexe au-dessus de $X$. Alors l'application $c$ de $X$ dans $X$ se relève en un isomorphisme $\mathbb{C}$-antilinéaire de fibrés $\hat{c}$ : $E \rightarrow E$ si et seulement si $\left(c^{*} E\right)^{*}=E$.

Le problème est qu'il n'existe pas toujours une involution $\mathbb{C}$-antilinéaire qui relève $c$, voir par exemple [4].

Définition 2.3. Un fibré $E$ est dit $c$-réel s'il existe une involution antilinéaire de $E$ dans $E$ relevant $c$. Dans ce cas, on désigne par $\kappa: \Gamma(E) \rightarrow$ $\Gamma(E)$ l'involution sur l'espace des sections de $E$ induite par $\hat{c}$ :

$$
\kappa(s)=\hat{c}^{-1} \circ s \circ c .
$$

Une section $s$ de $E$ est dite symétrique si $\kappa(s)=s$.

Lemme 2.4. Soit $L$ un fibré en droites vérifiant $L=\left(c^{*} L\right)^{*}$. Alors $L^{2}$ est c-réel.

Démonstration. Soit $\hat{c}$ une application antilinéaire de $L$ dans $L$ relevant c. L'application $\hat{c} \circ \hat{c}$ est un isomorphisme linéaire de $L$ dans $L$ relevant l'identité. Il existe donc une fonction complexe $a: X \rightarrow \mathbb{C}^{*}$ telle que $\hat{c} \circ$ $\hat{c}(x, \lambda)=(x, a(x) \lambda)$. En utilisant l'égalité $\hat{c}^{2} \circ \hat{c}=\hat{c} \circ \hat{c}^{2}$, on constate que cette fonction $a$ vérifie l'identité:

$$
a=\overline{a(c)} \text {. }
$$

Si l'on veut construire un nouveau relèvement de $c$, il suffit de diviser $\hat{c}$ par une application complexe ne s'annulant pas: $\hat{c}_{\phi}=\frac{1}{\phi} \hat{c}$. Ainsi, $\hat{c}_{\phi}^{2}=\hat{c}^{2} \frac{1}{\phi \overline{\phi(c)}}$. Maintenant, il est facile de vérifier que l'application

$$
\hat{c}_{L^{2}}=\frac{1}{a} \hat{c} \otimes \hat{c}
$$

est une involution antilinéaire de $L^{2}$ dans $L^{2}$ relevant $c$.

Fait: Soit $\left(X^{2 n}, J\right)$ une variété presque complexe munie d'une involution $J$-antiholomorphe. Alors pour tout $p$ et $q$ inférieurs à $n$, le fibré des $(p, q)$-formes est $c$-réel, et ont peut choisir $\kappa(\beta)=\overline{c^{*} \beta}$.

Supposons que la partie réelle $\mathcal{L}=\mathbb{R} X$ soit non vide. Dans ce cas, il est facile de démontrer que $\mathcal{L}$ est une sous-variété lagrangienne. Par conséquent, la restriction du fibré en droites complexes $L$ à $\mathcal{L}$ est topologiquement triviale puisque sa classe de Chern $\left[\omega_{\mid \mathcal{L}}\right]$ est nulle. Quitte à remplacer $L$ par une 
certaine puissance $L^{N}$, il existe donc une section $e$ de $L$ ne s'annulant pas sur un voisinage $V$ de $\mathcal{L}$. Ceci nous permet de choisir une involution de $L$ particulière:

Lemme 2.5. Si $\mathbb{R} X$ est non vide, et quitte à remplacer $L$ par une puissance paire $L^{2 N}$, pour toute section trivialisante e de $L$ sur le voisinage $V$ de $\mathbb{R} X$, de norme 1 sur $V$, on peut choisir $\hat{c}$ de sorte que $\hat{c}(e)=e$ aux points de $\mathbb{R} X$.

Démonstration. Soit $f \in C^{\infty}\left(V, S^{1}\right)$, telle que $\hat{c}(e)=f e$ sur $V$. Etendons à $X$ cette application. Quitte à considérer $L^{2}$ au lieu de $L$, l'application $f f(c)$ admet une racine carrée $g$ qu'on peut choisir égale à $f$ sur $\mathbb{R} X$ et vérifiant $g(c)=g$. Maintenant, le nouveau morphisme de fibré $\tilde{c}=\frac{1}{g} \hat{c}$ est l'identité au-dessus de $\mathbb{R} X$.

2.1.1. Connexions réelles. On suppose dorénavant que $L$ est un fibré $c$-réel. Sur $L$ on fixe une métrique hermitienne invariante par $c$, qui existe toujours. L'involution $\kappa$ s'étend à $\Gamma\left(T X^{*} \otimes L\right)$ par:

$$
\kappa: \alpha \otimes \lambda \mapsto \overline{c^{*} \alpha} \otimes \kappa(\lambda)
$$

Si $\nabla$ est une connection hermitienne, $\nabla s \in \Gamma\left(T X^{*} \otimes L\right)$, ce qui nous permet de construire une involution naturelle sur les connexions de $L$ par

$$
\kappa(\nabla)=\kappa \nabla \kappa .
$$

Il est alors aisé de démontrer le lemme suivant:

Lemme 2.6. Si $\nabla$ est une connection hermitienne de courbure -i $\omega$, alors $\kappa(\nabla)$ également. De plus pour toute connexion $\kappa$-invariante, et toute section $s$ de $L$, on $a|\nabla \kappa s|=|\nabla s|(c)$, de même que $\left|\nabla^{0,1} \kappa(s)\right|=\left|\nabla^{0,1} s\right|(c)$.

On a par ailleurs le

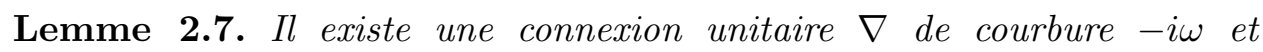
$\kappa$-invariante sur $X$.

Démonstration. Si $\nabla_{0}$ est une connexion hermitienne de courbure $-i \omega$, alors $\nabla=\frac{1}{2}\left(\nabla_{0}+\kappa\left(\nabla_{0}\right)\right)$ convient.

Dans toute la suite, on munira notre fibré d'une telle connexion.

2.2. Le théorème de Donaldson $[\mathbf{1}]$. Soit $(X, \omega, J)$ une variété symplectique compacte munie d'une structure presque complexe $J$ compatible avec $\omega$, c'est-à-dire telle que $\omega(\cdot, J$.$) est une métrique riemanienne. Si \omega$ est à périodes entières, il existe un fibré en droites complexes $L$ sur $X$, dont la classe de Chern représente la classe $[\omega] \in H^{2}(X, \mathbb{Z})$. Si l'on muni $L$ d'une norme hermitienne, il existe une connexion unitaire $\nabla$ sur $L$ de courbure $-i \omega$. Tous ces objets ont une extension naturelle pour chaque puissance $L^{k}$ du fibré. En particulier, on notera $g_{k}$ la métrique $k g$. 
Définition 2.8. Soit $E$ un fibré hermitien sur $X$. Une suite $\left(s_{k}\right)_{k}$ de sections de $E \otimes L^{k}$ est asymptotiquement holomorphe (AH) s'il existe une constante $C$ indépendante de $k$, telle que pour tout $k,\left|\nabla^{0,1} s_{k}\right| \leq C$, telle que sa dérivée est en norme inférieure à $C \sqrt{k}$, et sa dérivée seconde en est de norme inférieure à $C k$. La suite $\left(s_{k}\right)_{k}$ est de plus $\epsilon$-transverse sur $X$ s'il existe $\epsilon>0$, tel que pour tout $x$ tel que $\left|s_{k}(x)\right| \leq \epsilon, \nabla s_{k}(x)$ admet un inverse à droite de norme inférieure à $(\eta \sqrt{k})^{-1}$.

Le théorème principal de $[\mathbf{1}]$, originellement démontré si $E=X \times \mathbb{C}$, et généralisé par Auroux pour le cas général, est le suivant:

Théorème $2.9([\mathbf{1}, \mathbf{5}])$. Soit $E$ un fibré hermitien sur $X$. Pour toute suite de sections $\left(s_{k}\right)_{k} A H$ de $E \otimes L^{k}$, et pour tout $\epsilon>0$ fixé, alors il existe un $\eta>0$, une suite de sections $\left(\tilde{s}_{k}\right)_{k} A H, \eta$-transverse pour $k$ assez grand, avec $\left|s_{k}-\tilde{s}_{k}\right| \leq \epsilon$, et $\left|\nabla s_{k}-\nabla \tilde{s}_{k}\right| \leq \epsilon \sqrt{k}$.

Il nous faut rappeler les idées de la démonstration de ce théorème, dans le cas où $E$ est le fibré en droites complexes trivial. Pour tout réel $D>0$ a priori fixé, il existe un recouvrement par "couleurs," c'est-à-dire par des ensembles de boules de $g_{k}$-taille égale à 1 et dont les centres sont $g_{k}$-distants d'au moins $D$. Le nombre $N$ de ces couleurs augmente évidemment avec $D$, mais pas avec $k$. On perturbe en $N$ étapes la suite de sections $s_{k}$ par une somme pondérée de sections essentiellement localisées au-dessus des centres des boules d'une couleur donnée. Plus précisément, ces sections sont du même modèle:

Lemme 2.10. Soit $x \in X$. Il existe une suite de sections $\left(\sigma_{k, x}\right)_{k}$ asymptotiquement holomorphe satisfaisant les inégalités suivantes:

(1) $\left|\sigma_{k, x}\right| \geq \frac{1}{2}$ sur $B_{g_{k}}(x, 1)$;

(2) $\left|\sigma_{k, x}\right|_{C^{2}} \leq p\left(d_{k}(x, y)\right) e^{-d_{k}(x, y)^{2}}$;

(3) $\sigma_{k, x}$ a un support inclus dans $B_{g_{k}}\left(x, k^{1 / 6}\right)=B_{g}\left(x, k^{-1 / 3}\right)$, où $p$ est un polynôme indépendant de $k$.

La perturbation sur une couleur est faite de la façon suivante:

$$
s_{k} \rightarrow s_{k}+\sum_{x_{i} \in \text { couleur }} w_{i} \sigma_{k, x_{i}} \text {. }
$$

A chaque étape, un théorème de Sard permet de choisir les $w_{i}$ de façon à ce que la nouvelle section devienne $\eta$-transverse sur la nouvelle couleur et le reste sur les anciennes, mais pour un $\eta$ de plus en plus petit. Le théorème de Sard utilisé est quantitatif, ce qui permet d'effectuer cette récurrence en un nombre d'étapes ne dépendant pas de $k$.

2.3. Construction de l'hypersurface réelle. La plupart des outils techniques qui nous permettent d'obtenir le Théorème 1.1 sont partiellement 
présents dans les articles $[\mathbf{1}, \mathbf{2}, \mathbf{5}, \mathbf{6}]$. Dans ce dernier article il est démontré que si $\mathcal{L}$ est une sous-variété lagrangienne munie d'une fonction de Morse réelle, alors il existe un pinceau de Lefschetz $F$ à la Donaldson, tel qu'une isotopie de $\mathcal{L}$ se projette par $F$ sur un arc réel. Pour démontrer ce résultat, les auteurs ont besoin de considérer $\mathcal{L}$ comme la partie réelle d'une stucture réelle semi-locale, et de construire les perturbations de façon symétriques par rapport à cette structure au voisinage de $\mathcal{L}$. Dans notre cas, nous devons construire les sections et leur perturbation de façon symétrique, mais cette fois globalement sur tout $X$. Par ailleurs, nous aimerions transversaliser n'importe quelle suite de sections $\mathrm{AH}$ symétrique, pas nécessairement à partir d'une fonction de Morse sur $\mathbb{R} X$. Nous allons en fait démontrer la proposition suivante.

Proposition 2.11. Soit $E$ un fibré hermitien c-réel, et $\left(s_{k}\right)_{k}$ une suite de sections symétriques et $A H$ de $E \otimes L^{k}$. Alors il est possible de la perturber en une suite de sections symétriques, $A H$ et transverse sur $X$.

Remarquons que le Théorème 1.1 est une conséquence immédiate de cette proposition, avec $E=X \times \mathbb{C}$.

Fait: on peut toujours choisir le réseau de boules, ainsi que chaque couleur, invariante par $c$. De plus, on peut faire en sorte que si l'une de ces boules rencontre $\mathbb{R} X$, alors la boule est invariante par $c$.

Par souci de clarté, nous démontrons le théorème pour $E=X \times \mathbb{C}$, puis nous expliquerons les adaptations nécessaires pour le cas général.

Démonstration dans la cas où $E=X \times \mathbb{C}$. Désignons par $\Lambda^{\prime}\left(\right.$ resp. $I_{i}^{\prime}$ ) un sous-réseau minimal (resp. de la couleur $I_{i}$ ) tel que

$$
\left.\bigcup_{x \in \Lambda^{\prime}} B_{g_{k}}(x, 1) \cup B_{g_{k}}(c(x)), 1\right)=X
$$

(resp. pour la couleur $i$, mutatis mutandis). Lors de la transversalisation sur une boule de la couleur $I_{i}$, si l'on perturbe la section $s_{k}$ par $w \sigma_{k, p}$, on perd le caractère réel. Nous avons donc besoin d'un raffinement du théorème de Sard quantitatif présent dans [1]:

Proposition 2.12. Il existe un entier $p$ et $\delta_{0}>0$, tels que pour tout $0<$ $\delta<\delta_{0}$, si $\sigma=\delta \log \left(\delta^{-1}\right)^{-p}$, et $f$ une fonction complexe définie sur la boule $B\left(0, \frac{11}{10}\right) \subset \mathbb{C}^{n}$ vérifiant

$$
|f| \leq 1, \quad|\bar{\partial} f|_{C^{1}} \leq \sigma,
$$

alors il existe une constante $w \in \mathbb{R}$, avec $|w| \leq \delta$, et $f-w$ est $\sigma$-transverse sur la boule unité de $\mathbb{C}^{n}$. 
Remarque. Notons que la seule différence avec les propositions de [1] est que l'on peut choisir $w$ réel.

Pour poursuivre, nous avons besoin également du raffinement suivant du Lemme 2.10, qui nous permet de remplacer $\sigma_{k, x}$ par une section $\mathrm{AH}$ symétrique:

Fait: Pour tout $x \in \Lambda^{\prime}$, la section $\mathrm{AH}$ et symétrique

$$
\hat{\sigma}_{k, x}=\frac{\sigma_{k, x}+\kappa\left(\sigma_{k, x}\right)}{2}
$$

est de norme uniformément minorée sur $B_{g_{k}}(x, 1) \cup B_{g_{k}}(c(x), 1)$.

On applique donc le procédé de Donaldson sur les boules centrées sur les points $x$ de $\Lambda^{\prime}$, mais en utilisant la Proposition 2.12 appliquée à

$$
f=\frac{s_{k}}{\hat{\sigma}_{k, x}}
$$

ce qui permet de trouver un $w$ rel de sorte que $s_{k}+w \hat{\sigma}_{k, x}$ est symétrique et transverse sur la boule $B_{g_{k}}(x, 1)$. On a alors par le Lemme 2.6 la transversalisation automatique sur les boules centrées sur les points de $c\left(\Lambda^{\prime}\right)$, ce qui démontre le Théorème 1.1.

Nous donnons maintenant une démonstration du raffinement du lemme de Sard.

Démonstration de la Proposition 2.12. La preuve suit jusqu'au dernier moment la preuve classique donnée par $[\mathbf{1}]$. L'application $f$ vérifiant $|\bar{\partial} f| \leq \sigma$ peut être $C^{1}$-approchée par une application holomorphe $\tilde{f}$ sur la boule unité, et l'erreur est inférieure à un multiple (borné) de $\sigma$. Ensuite il est possible de $C^{1}$-approcher $\tilde{f}$ de $\sigma$ par un polynôme complexe $g$ de degré inférieur ou égal à $C \log \left(\delta^{-1}\right)$, où $C$ est une constante ne dépendant que de la boule. Pour toute fonction complexe $h$, soit

$$
Y_{h, \epsilon}=\left\{z \in \mathbb{B}^{2 n},\left|d_{x} h\right| \leq \epsilon\right\},
$$

et $Z_{h, \epsilon}$ le $\epsilon$-voisinage tubulaire de $f\left(Y_{h, \epsilon}\right)$. Nous avons l'inclusion: $Z_{f, \sigma} \subset$ $Z_{g, c \sigma}$, où $c$ est une constante de structure. Si l'on trouve un réel $w$ dans un disque de taille $\delta$ évitant $Z(g, c \sigma)$, alors on a prouvé la proposition. Rappelons la

Proposition 2.13. Soit $P$ un polynôme de $\mathbb{R}^{m}$ dans $\mathbb{R}$, tel que 1 soit une valeur régulière de $P$ sur $\mathbb{B}^{m}$, ainsi que de la restriction de $P$ sur $S^{m-1}$. Alors le nombre de composantes du sous-niveau $\{P \leq 1\}$ ainsi que leur diamètre (pour la métrique induite) sont p-bornés.

On dit qu'une quantité associée à un polynôme est $p$-bornée si elle est majorée par une puissance du degré du polynôme. Après une infime perturbation de $g$, appliquons la proposition précédente au polynôme réel $|\partial g / c \sigma|^{2}$, qui est de degré $C \log \left(\sigma^{-1}\right)$, où $C$ est une nouvelle constante de structure. 
Le diamètre pour la métrique ambiante de l'image d'une composante de $Y_{g, c \sigma}$ est majoré par $C^{\prime} \sigma \log \left(\sigma^{-1}\right)^{p}$, où $p$ est la puissance donnée par la proposition. Le voisinage $\sigma$-tubulaire de $Z_{g, c \sigma}$ est donc contenu dans une réunion de $\log \left(\sigma^{-1}\right)^{p}$ disques de rayons égaux à $\sigma \log \left(\sigma^{-1}\right)^{p}+\sigma$. Ce dernier ajout de $\sigma$ ne change rien, et on peut l'oublier. L'intersection de l'ensemble prohibé avec $\mathbb{R}$ recouvre donc au plus une longueur de $\sigma \log \left(\sigma^{-1}\right)^{2 p}$. Si le rayon du disque est par exemple $\sigma \log \left(\sigma^{-1}\right)^{3 p}$, il reste encore beaucoup de place pour trouver un $w$ réel dans le complémentaire de $Z_{g, c \sigma}$ dans $[-\delta, \delta]$. On obtient alors la proposition, en effectuant un changement de variables comme dans [1].

Démonstration de la Proposition 2.11 dans le cas où $E$ est un fibré hermitien quelconque. Nous suivons la démonstration de [5] et l'adaptons en cours de route à notre cas. Elle consiste à transversaliser $s_{k}$ composante par composante. Plus précisément, on choisit un recouvrement par des ouverts $U_{i}$ de trivialisation du fibré $E$, tels que $c\left(U_{i}\right)$ coïncide avec un autre $U_{j}$, et précisément $U_{i}$ si $U_{i}$ rencontre $\mathbb{R} X$. On transversalise sur la moitié de l'ensemble de ces paires d'ouverts en ajoutant à $s_{k}$ des sections symétriques, si bien qu'automatiquement, cela donnera la transversalisation sur le reste des ouverts. Supposons que sur un certain $U_{i}$, les $r$ premières composantes de $s_{k}$, qu'on écrit $\pi_{\leq r} s_{k}$, forment une section transverse, et définissent donc une sous-variété symplectique $W_{k, r}$ lisse sur $U_{i}$. Auroux montre qu' il est possible de transversaliser la restriction de la $r+1$-ième composante $\pi_{r+1} s_{k}$ sur $W_{k, r}$ en lui ajoutant une petite section $\mathrm{AH} \tau_{k, r}$. Il démontre ensuite que cela implique automatiquement que $\pi_{\leq r} s_{k} \oplus\left(\pi_{r+1} s_{k}+\tau_{r, k}\right)$ est en fait transverse sur tout $U_{i}$. Dans notre cas, nous savons que $W_{k, r}$ est invariant par $c$. Par ailleurs, puisque la section partielle $\pi_{\leq r} s_{k}$ est transverse, il est facile de voir qu'il existe un $\rho>0$ indépendant de $k$, tel que pour tout point $x \in \mathbb{R} X$, l'intersection de $B_{g_{k}}(x, \rho)$ avec $W_{k, r}$ est toujours ou bien connexe et de partie réelle non vide, ou bien est vide. Pour le procédé de Donaldson, on considère donc un réseau $\Lambda^{\prime}$ invariant par $c$, et centré sur des points de $W_{k, r}$, de boules $B_{g_{k}}(x, \rho)$. Il est alors possible d'appliquer la démonstration décrite dans [5], sans paramètre $t$, et en utilisant la Proposition 2.12 pour chaque transversalisation, et enfin $\hat{\sigma}_{k, x}$ au lieu de $\sigma_{k, x}$. Au total, on a ajouté des sections symétriques qu'on somme en une section $\tau_{k, r}$, de sorte que la section $\pi_{\leq r} s_{k} \oplus\left(\pi_{r+1} s_{k}+\tau_{r, k}\right)$ est symétrique et transverse sur $U_{i}$. Pour tout ouvert $U_{i}$ trivialisant $E$, on répète le processus, dont le nombre de pas ne dépend que de $E$ et $X$, si bien qu'au total, on obtient la Proposition 2.11 dans toute sa généralité.

2.4. Unicité. Nous démontrons maintenant une version d'unicité des hypersurfaces réelles construites:

Proposition 2.14. Soient $\left(s_{1, k}\right)_{k}$ et $\left(s_{2, k}\right)_{k}$ deux suites de sections de $E \otimes L^{k}$ $A H$, transverses et symétriques, et telles que $s_{1, k}(x)=s_{2, k}(x)$ pour tout 
$x \in \mathbb{R} X$. Alors pour tout $k$ assez grand, il existe une isotopie de sections $A H$ et transverses reliant les lieux d'annulations de $s_{1, k}$ et $s_{2, k}$.

Démonstration. Pour faciliter la lecture, nous démontrons cette proposition dans le cas où $E=X \times \mathbb{C}$. Tout comme dans [5], on considère la suite de sections symétriques $s_{t, k}=t s_{1, k}+(1-t) s_{2, k}$, et l'on va montrer qu'on peut transversaliser cette suite uniformément en $t$. Le problème principal est que la Proposition 2.12, contrairement à sa version plus souple de [1], n'admet pas de généralisation avec un paramètre. En effet, le lieu des $w$ réels interdits à chaque temps $t$ est certes très petit, mais sépare en général l'axe des réels en au moins deux parties, au sein desquelles il faut choisir $w_{t}$. Or l'une de ces parties peut disparaitre pour un certain $t$, si bien qu'il faudrait alors choisir des $w_{t}$ discontinus, ce qui n'est évidemment pas possible. Nous verrons plus bas que la condition d'égalité des deux sections aux points de $\mathbb{R} X$ permet de s'abstenir de cette version. En dehors d'un voisinage de $g$-taille fixe de $\mathbb{R} X$, nous pouvons utiliser la version paramétrique d'Auroux (Proposition 3 dans [5]), et additionner à $s_{t, k}$ la section $w_{t} \sigma_{k, x}+\overline{w_{t}} \kappa\left(\sigma_{k, x}\right)$. Cette dernière est symétrique, et puisque les supports de $\sigma_{k, x}$ et $\kappa\left(\sigma_{k, x}\right)$ sont disjoints, d'une part la transversalité aquise d'un côté, sur $B_{g_{k}}(x, 1)$, n'est pas perturbée par le terme $\overline{w_{t}} \kappa\left(\sigma_{k, x}\right)$, d'autre part par le Lemme 2.6, la transversalité est spontanément acquise de l'autre côté, sur $c\left(B_{g_{k}}(x, 1)\right)$ par ce dernier terme.

Maintenant, étudions la situation sur une boule $B_{g_{k}}(x, 1)$, avec $x \in \mathbb{R} X$. Après trivialisation par le Lemme 2.24 ci-dessous, nous utilisons le lemme suivant:

Lemme 2.15. Soit $f$ une fonction complexe définie sur $2 \mathbb{B}^{2 n}$, vérifiant $|\bar{\partial} f|_{C^{1}}<\epsilon$, et $f(x)=0$ pour tout $x$ dans $\mathbb{R}^{n}$. Alors $|f|_{C^{1}\left(\mathbb{B}^{2 n}\right)}<c \epsilon$, où $c$ est une constante indépendante de $f$ et de $\epsilon$.

Démonstration. Pour démontrer ce lemme, on commence par trouver grâce au Lemme 28 de $[\mathbf{1}]$ une fonction holomorphe $\tilde{f}$ sur $\frac{3}{2} \mathbb{B}^{2 n}$ telle que

$$
|f-\tilde{f}|_{C^{1}\left(\frac{3}{2} \mathbb{B}^{2 n}\right)}<K \epsilon
$$

où $K$ est une constante indépendante de $f$ et de $\epsilon$. Ensuite, le Lemme 27 de [1] nous donne un polynôme complexe $p$, tel que $|\tilde{f}-p|_{C^{1}}<\epsilon$. On a donc par la seconde hypothèse $\left|p_{\mathbb{R}^{n}}\right|_{C^{1}}<(K+1) \epsilon$, ce qui implique, puisque $p$ est complexe, l'existence d'une constante $c>0$ telle que $|p|_{C^{1}}<c(K+1) \epsilon$, et donc $|f|_{C^{1}}<(1+c)(K+1) \epsilon$.

En appliquant ce lemme à $f=\frac{s_{1, k}-s_{2, k}}{\sigma_{k, x}}$ via la traditionnelle renormalisation par $1 / \sqrt{k}$, on obtient que $s_{1, k}-s_{2, k}$ est en norme inférieure à $C / \sqrt{k}$, et sa dérivée covariante inférieure en norme à $C$ (pour la métrique $g$ ). C'est donc également vrai pour $s_{t, k}-s_{2, k}$ uniformément en $t$, et puisque $s_{2, k}$ est transverse sur $B_{g_{k}}(x, 1)$, la section $s_{t, k}$ l'est aussi, pour $k$ assez grand. 
Il reste maintenant à transversaliser $s_{t, k}$ dans l'espace entre les deux régions précédentes. Pour cela, il nous faut un raffinement de la Proposition 3 de [5], qui est donné par la proposition suivante de [6]:

Proposition 2.16. [6]. Soit $C>0, \epsilon>0$. Alors il existe un entier $p$ et une constante $\delta_{0}$, tels que pour tout $0<\delta<\delta_{0}$, si $\sigma=\delta\left(\log \left(\delta^{-1}\right)\right)^{-p}$ et $f_{t}$, $h_{t}$ deux chemins continus paramétrés par $[0,1]$ de fonctions complexes sur la boule $B\left(0, \frac{11}{10}\right) \subset \mathbb{C}^{n}$ vérifiant les majorations suivantes:

$$
\left|f_{t}\right| \leq 1, \quad\left|\bar{\partial} f_{t}\right|_{C^{1}} \leq \sigma, \quad\left|h_{t}\right| \leq 1-\epsilon, \quad\left|d h_{t}\right| \leq C, \quad\left|\bar{\partial} h_{t}\right|_{C^{1}} \leq \sigma .
$$

Alors il existe un chemin continu $w_{t} \in \mathbb{C}$ vérifiant $\left|w_{t}\right|<\delta$, tel que $f_{t}-w_{t}-$ $\bar{w}_{t} h_{t}$ soit $\sigma$-transverse à 0 sur la boule unité de $\mathbb{C}^{n}$.

Maintenant, il suffit d'ajouter à $s_{t, k}$ la section $w_{t} \sigma_{k, x}+\bar{w}_{t} \kappa\left(\sigma_{k, x}\right)$, où $w_{t}$ est déterminée par la proposition précédente. En effet, la section $s_{t, k}-w_{t} \sigma_{k, x}-$ $\bar{w}_{t} \kappa\left(\sigma_{k, x}\right)$ correspond à la fonction

$$
f_{t}=\frac{s_{t, k}}{\sigma_{k, x}}-w_{t}-\bar{w}_{t} \frac{\kappa\left(\sigma_{k, x}\right)}{\sigma_{k, x}} .
$$

Quitte à prendre des boules de trivialisations assez petites (de $g_{k}$-taille indépendantes de la boule et de $k$ ), les deux points $x$ et $c(x)$ sont à une $g_{k}$-distance d'au moins 2, si bien que la fonction $h_{t}=h=\frac{\kappa\left(\sigma_{k, x}\right)}{\sigma_{k, x}}$ et $f_{t}$ vérifient les hypothèses de la proposition.

2.5. La partie réelle de l'hypersurface symplectique. Dans ce paragraphe, on suppose que la partie réelle est non vide. Le but est de construire des hypersurfaces réelles symplectiques dont le lieu réel est ou bien vide, ou bien non vide, et dans ce cas de maximiser si possible sa topologie. Nous pouvons énoncer deux propositions simples. Le premier cas est minimal, puisque la partie réelle est vide:

Proposition 2.17. Il existe une hypersurface symplectique de partie réelle vide.

Démonstration. Dans [7], les auteurs ont construit une section AH de norme uniformément minorée au-dessus d'une sous-variété lagrangienne $\mathcal{L}$ donnée. Ce théorème est raffiné dans le Lemme 5.4 de [6], où la section est cette fois localement symétrique au voisinage de $\mathcal{L}$ si celle-ci est localement la partie réelle d'un involution antisymplectique, et s'annule au-delà de ce voisinage, ce qui convient à notre situation. Il suffit alors de transversaliser par notre Proposition 2.11 pour obtenir une section symétrique et ne s'annulant pas sur $\mathbb{R} X$.

La proposition suivante cherche, au contraire, à maximiser la topologie de la partie réelle de l'hypersurface: 
Théorème 2.18. Soit $\left(X^{2 n}, \omega, J, c\right)$ une variété symplectique réelle de partie réelle non vide. Si $[\omega]$ est rationnelle, il existe un $\epsilon>0$ tel que pour tout $k$ assez grand, il existe une hypersurface symplectique réelle Poincaré duale à $2 k[\omega]$ et une suite de sections $A H\left(s_{k}\right)_{k}$, tel que le nombre de composantes connexes de sa partie réelle est au moins $\epsilon k^{n / 2}$.

Remarque 2.19. Rappelons que dans [1], il est démontré que les hypersurfaces sont toujours connexes pour $n \geq 2$.

Remarque 2.20. En dimension 4, notre théorème donne une minoration en $\epsilon k$, ce qui est à comparer avec le théorème d'Harnack, qui majore dans $\mathbb{C} P^{2}$ complexe le nombre d'ovales d'une courbe holomorphe réelle par une borne de l'ordre de $k^{2} / 2$.

Remarque 2.21. Les hypersurfaces de Donaldson tendent à remplir tout l'espace, et donc n'ont pas de raison particulière de se concentrer sur la partie réelle de $X$. On a en effet [1, Proposition 40]:

$$
\frac{1}{k} Z_{k} \rightarrow \omega
$$

en tant que courants. On peut donc s'attendre, dans notre cas, à ce que la partie réelle de ces hypersurfaces soit relativement représentative de celle du lieu d'annulation d'une section prise au hasard.

Remarque 2.22. Dans [8], les auteurs démontrent que dans $\mathbb{C} P^{1}$ et pour une certaine mesure naturelle sur les polynômes de degré $k$, le nombre moyen de racines réelles d'un polynôme réel est $\sqrt{k}$, ce qui est précisément notre cas à une constante près.

La proposition suivante montre que de toutes façons il ne fallait pas s'attendre à obtenir vraiment mieux que ce qu'offre le théorème précédent:

Proposition 2.23. Soit $\left(X^{2 n}, \omega, J, c\right)$ une variété symplectique réelle de partie réelle non vide, et $\left(s_{k}\right)_{k}$ une suite de sections $A H$, uniformément transverse et symétrique. Alors il existe une constante $C$ indépendante de $k$, telle que le nombre de composantes de la partie réelle de $s_{k}^{-1}(0)$ n'excède pas $C k^{n / 2}$.

Démonstration. Soit $x$ un zéro quelconque de $s_{k}$. Puisque $s_{k}$ est transverse, il existe un $\epsilon>0$ tel qu'en $x,\left|\nabla s_{k}\right| \geq 2 \epsilon \sqrt{k}$. Par ailleurs $\nabla \nabla s_{k}$ est un $O(k)$, si bien que pour $\eta$ assez petit indépendant de $k$, la dérivée reste en norme supérieure à $\epsilon \sqrt{k}$ sur la boule $B_{g_{k}}(x, \eta)$. Il s'ensuit (cf. [5], partie 3.3) que sur cette boule, l'hypersurface est triviale. Par conséquent, chaque composante connexe de $s_{k}^{-1}(0) \cap \mathbb{R} X$ contient une boule de rayon $\eta / \sqrt{k}$, telle que deux de ces boules ne s'intersectent pas. Si $N$ est le nombre de ces composantes, $N(\eta / \sqrt{k})^{n}$ est donc majoré par le volume de $\mathbb{R} X$, ce qui implique la contrainte $N \leq C k^{n / 2}$ pour une constante $C$ indépendante de $k$. 
Démonstration du Théorème 2.18. Nous avons d'abord besoin du lemme suivant:

Lemme 2.24. Soit $x \in \mathcal{L}$. Alors il existe une application $\phi: B_{g}(x) \rightarrow \mathbb{C}^{n}$ vérifiant:

(i) $\phi(x)=0$;

(ii) $\phi^{*} \omega_{0}=\omega$;

(iii) $\phi(\mathcal{L})=\mathbb{R}^{n}$

(iv) $\phi^{*} J_{0 \mid \mathcal{L}}=J_{\mid \mathcal{L}}$

(v) $\phi$ se relève en un isomorphisme entre $L$ et le fibré trivial sur $\mathbb{C}^{n}$;

(vi) $\tilde{c}=\phi_{*} c$, où $c_{0}$ est la conjugaison sur $\mathbb{C}^{n}$ et vérifie $d \tilde{c}=d c$ aux points de $\mathcal{L}$.

Démonstration du lemme. Les cinq premiéres propriétés sont classiques (cf. $[6])$. Quant à la dernière, remarquons d'abord que $d c_{\mid T \mathcal{L}}=d \tilde{c}_{\mid T \mathcal{L}}$. Ensuite, si $\lambda$ est un vecteur normal à $T \mathcal{L}$, alors $J \lambda \in T \mathcal{L}$, et donc

$$
d \tilde{c}(\lambda)=-d \tilde{c}\left(J^{2} \lambda\right)=J d \tilde{c}(J \lambda)=J^{2} \lambda=-\lambda=d c(\lambda)
$$

ce qui démontre le (vi).

L'idée est de prendre sur $\mathbb{R} X$ un réseau dont la maille est de $g_{k}$-taille $D$, la constante $D$ étant indépendante de $k$, puis pour chaque sommet $x_{i}$, de construire une suite de sections $\tau_{i, k}$ suffisamment transverse, et dont la partie réelle du lieu d'annulation est localement et approximativement une hypersphère incluse dans $B_{g_{k}}\left(x_{i}, 1\right)$. Ce qui suit traite en fait d'une situation plus générale. Soit $f: \mathbb{B}^{2 n} \rightarrow \mathbb{C}$ une fonction holomorphe, non singulière, vérifiant $\overline{f(\bar{z})}=f(z)$ pour tout $z \in 2 \mathbb{B}^{2 n}$, et telle que la partie réelle de son lieu d'annulation soit incluse dans la boule ouverte. L'exemple dont on se servira est

$$
f\left(z_{1}, \ldots, z_{n}\right)=z_{1}^{2}+\cdots+z_{n}^{2}-\frac{1}{2} .
$$

Dans ce modèle local, la suite de sections

$$
\tau_{k}(z)=f(z \sqrt{k}) e^{-k|z|^{2}}
$$

est holomorphe et symétrique. Elle est de plus uniformément $\epsilon$-transverse pour un $\epsilon$ donné sur la boule. En effet, remarquons d'abord le fait suivant:

$$
\exists \eta>0, \quad|f|<\frac{\eta}{2 e C} \Longrightarrow|d f|>\eta,
$$

où $C$ est elle que $\left|\nabla e^{-k|z|^{2}}\right| \leq C \sqrt{k}$. Si bien que si $\left|\tau_{k}\right|<\eta / 2 e^{2} C$, alors

$$
\left|\nabla \tau_{k}\right| \geq \sqrt{k}|d f| e^{-k|z|^{2}}-|f|\left|\nabla e^{-k|z|^{2}}\right| \geq \sqrt{k} \eta e^{-1} / 2
$$


ce qui démontre la transversalité uniforme de $\tau_{k}$. De plus, il est clair que la dérivée de $\tau_{k}$ est en norme inférieure à $C^{\prime} \sqrt{k}$, où $C^{\prime}$ ne dépend pas non plus de $k$. Enfin, la restriction à $\mathbb{R}^{n}$ de la suite de sections est également transverse, pour les mêmes raisons que précédemment.

Nous utilisons le lemme précédent afin de rapatrier cette suite de sections sur notre variété symplectique. Nous noterons $\tau_{i, k}$ la section $\phi_{i}^{*} \tau_{k}$, où $\phi_{i}$ est le difféomorphisme du lemme, centré sur le point $x_{i}$. La suite de sections obtenue est $\mathrm{AH}$, symétrique, et $\epsilon$-transverse pour un $\epsilon$ qu'on peut choisir uniforme pour tout point du réseau puisque la partie réelle $\mathbb{R} X$ est compacte. Le procédé de cut-off traditionnel chez Donaldson ne vient pas perturber ces propriétés. En revanche, la transversalité n'est plus vraie que sur une boule qu'on peut choisir de $g_{k}$-rayon 1 .

Maintenant, nous faisons la somme de toutes ces sections. Si la maille $D$ $\mathrm{du}$ réseau est suffisamment grande, mais indépendante de $k$, la somme de toutes les autres contributions laisse transverse chacune des sections particulières. En effet, la norme $C^{0}$ (resp. $C^{1}$ ) de la somme de toutes les autres contributions que $\tau_{i, k}$ est majorée par:

$$
\begin{gathered}
\left|\sum_{x_{j} \in \text { Réseau } \backslash\left\{x_{i}\right\}} \tau_{x_{j}, k}\right| \leq C e^{-D} \\
\sum_{x_{j} \in \text { Réseau } \backslash\left\{x_{i}\right\}} \nabla \tau_{x_{j}, k} \mid
\end{gathered}
$$

Au total, pour $D$ assez grand (indépendant de $k$ ) la transversalité d'origine au point $x_{i}$ est préservée par les ajouts des autres fonctions $\tau$ sur la boule $B_{g_{k}}\left(x_{i}, 1\right)$. La section continue donc à s'annuler sur $\mathbb{R} X$ sur une sous-variété isotope à la réunion des hypersphères, et ce dans un voisinage tubulaire de celles-ci et de $g_{k}$-taille de l'ordre de $e^{-D} / \epsilon$, bien inférieure à celle qui sépare $x$ des autres points du réseau, si l'on choisit $D$ assez grand. On a donc créé autant d'hypersurfaces (déformées des hypersphères) d'annulation que de points du réseau, soit $\epsilon^{\prime} \sqrt{k}$, où $\epsilon^{\prime}$ est une constante assez petite ne dépendant que de la géométrie de $(X, \omega)$. Enfin, la Proposition 2.11 nous permet de perturber $s_{k}$ en une suite de sections transverses et symétriques. Si la perturbation est assez faible, les pseudo-hypersphères réelles ne sont pas détruites, et le théorème est démontré.

2.6. Le cas intégrable. Démonstration de la Proposition 1.3. La Proposition 1.3 est déduite de la conjonction des résultats précédents, et du fait fondamental que lorsque $J$ est intégrable, c'est-à-dire que $X$ est une variété Kähler, il est possible (cf. [1, Proposition 34]) de rendre les sections concentrées du Lemme 2.10 holomorphes. Par ailleurs il est clair que si $s$ est une section holomorphe, $\kappa(s)$ est également holomorphe. Dans notre cas, il est 
crucial de trouver, pour tout point $p$ de $\mathbb{R} X$, une section symétrique concentrée et holomorphe du type prédédent. Il suffit comme dans la partie 1.3 de changer la phase de $\sigma_{k, p}$, puis de prendre $\frac{1}{2}\left(\sigma_{k, p}+\kappa\left(\sigma_{k, p}\right)\right)$, qui conserve toutes les propriétés souhaitées. En utilisant la version holomorphe de ces sections concentrées, on obtient tous les résultats précédents dans le cadre complexe.

\section{Pinceaux de Lefschetz réels}

L'existence de pinceaux de Lefschetz réels se fait en perturbant une paire de section AH. La proposition suivante (cf. Définition 5 de [2] et Proposition 5.5 de $[\mathbf{6}])$ représente la première étape à réaliser avant le théorème:

Proposition 3.1. Soient $s_{0}$ et $s_{1}$ des suites de sections $A H$ et symétriques. Alors pour $\epsilon>0$ assez petit, il existe une suite de sections $\tau_{0} \oplus \tau_{1}$ de $L^{k} \oplus L^{k}$ symétriques, telle que:

1. La section $s_{0}+\tau_{0}$ est $\epsilon$-transverse.

2. La section $\left(s_{0}+\tau_{0}\right) \oplus\left(s_{1}+\tau_{1}\right)$ est $\epsilon$-transverse,

3. La (1,0)-dérivée $\partial F$ de la fonction complexe $F=\left(s_{1}+\tau_{1}\right) /\left(s_{0}+\tau_{0}\right)$, est $\epsilon$-transverse sur l'ensemble $Z_{k, \epsilon}=\left\{\left|s_{0}+\tau_{0}\right| \geq \epsilon\right\}$.

4. $F(c)=\bar{F}$.

3.1. De la Proposition 3.1 au Théorème 1.6. Dans ce paragraphe, nous supposons que la proposition précédente est vraie, et nous démontrons sous cette hypothèse que chacune des conditions de la Définition 1.4 est vérifiée.

3.1.1. Propriété $(\boldsymbol{i v})$. Nous devons perturber les suites de sections $s_{0}$ et $s_{1}$ afin de satisfaire à la condition de la propriété (iv) de la Définition 1.4. Cette perturbation est réalisée dans [2], pp. 214-215, Lemme 11. Comme d'habitude, nous devons nous assurer que nous pouvons la réaliser de façon symétrique. Rappelons le principe de [2]. En un point $x$ de $N=\left\{s_{0}=s_{1}=\right.$ $0\}$, l'espace $T_{x} N$ est le noyau de l'opérateur:

$$
D_{x}:=\nabla s_{0} \oplus \nabla s_{1}: T_{x} X \rightarrow L_{x}^{k} \oplus L_{x}^{k} .
$$

Soit $N_{0}$ le supplémentaire symplectique de $T_{x} N$ dans $T_{x} X$. L'opérateur $D_{x}$ établit un isomorphisme (réel) entre $N_{0}$ et $L_{x}^{k} \oplus L_{x}^{k}$, et induit ainsi par tiré en arrière une structure complexe $j(D)$ sur $N_{0}$.

Lemme 3.2 (Lemme 11 de $[\mathbf{2}]$ ). Pour tout point $x$ dans $N, F=s_{1} / s_{0}$ peut être représentée sous la forme (iv) de la Définition 1.4 si et seulement si la restriction de la forme symplectique $\omega$ à $N_{0}$ est une forme $(1,1)$ pour $j(D)$ et positive.

Nous démontrons maintenant le lemme suivant: 
Proposition 3.3. Il existe une perturbation de $s_{0} \oplus s_{1}$ de sorte que $F$ soit symétrique et vérifie les conditions du lemme précédent.

Démonstration. La démonstration de la proposition se fait en deux temps. D'une part il nous faut trouver une structure complexe $j$ sur chaque fibre $N_{0}$ symétrique par rapport à $c$, telle que $\omega_{\mid N_{0}}$ soit $(1,1)$ pour $j$. D'autre part, un théorème d'inversion locale nous permet de perturber symétriquement les sections $s=s_{0} \oplus s_{1}$ en $\tilde{s}$ de sorte que $j=j(D(\tilde{s}))$. Soit

$$
\pi: T X \rightarrow N_{0}
$$

la projection symplectique sur $N_{0}$. Par définition de $N_{0}, g$, $\omega$ et $J$, l'endomorphisme $\pi J: N_{0} \rightarrow N_{0}$ vérifie

$$
g(u, v)=\omega(u, \pi J v) \quad \forall u \in N_{0}, \forall v \in N_{0} .
$$

Si $\pi J$ n'est pas tout à fait une structure complexe, ce n'est pas loin:

$$
(\pi J)^{2}=-I d_{\mid N_{0}}+O(1 / \sqrt{k}) .
$$

En effet, $N_{0}$ et $T N$ sont approximativement des variétés $J$-complexes, à $C / \sqrt{k}$ près, ce qui implique que

$$
|J-\pi J| \leq C / \sqrt{k},
$$

et donc aussi l'estimation précédente. De plus, on peut perturber $a=\pi J$ en une authentique structure complexe $j$ sur $N_{0}$, par la méthode classique suivante. Il est clair que $a$ est antiautoadjoint relativement à la métrique $g_{\mid N_{0}}$. Soit $q$ l'endomorphisme défini comme la racine carrée de $-a a^{*}$. L'application $j=a q^{-1}$ est alors une structure complexe compatible avec $\omega_{\mid N_{0}}$. L'estimation précédente montre que

$$
|\pi J-j| \leq C / \sqrt{k} .
$$

Enfin, chacun des éléments étant (anti)covariants par $d c$, le résultat est également covariant par $d c$, i.e $c^{*} j=-j$.

Montrons maintenant que cette structure est atteinte par l'intermédiaire d'une perturbation des sections de départ. Pour cela, soit $\mathcal{J}_{0}$ l'ensemble des structures complexes sur $N_{0}$. l'application:

$$
\begin{aligned}
j: G l\left(N_{0}, L^{k} \oplus L^{k}\right) & \rightarrow \mathcal{J}_{0} \\
D & \mapsto D^{-1} i D,
\end{aligned}
$$

où $i$ est la structure complexe sur $L^{k} \oplus L^{k}$. Puisque $s_{0} \oplus s_{1}$ est asymptotiquement holomorphe, on a

$$
\| j(\nabla s))-\pi J \| \leq C\left|(\nabla s)^{-1}\right| \leq C /(\eta \sqrt{k}),
$$

puisque la norme de l'inverse de $D_{x}$ est inférieure à $1 /(\eta \sqrt{k})$, en vertu de la $\eta$-transversalité de la section $s=s_{0} \oplus s_{1}$, et que $s$ est $\mathrm{AH}$, donc son $\bar{\partial}$ est 
inférieur en norme à $C$. La différentielle de $j$ en $D$ est

$$
d_{D} j(H)=-D^{-1} H D^{-1} i D+D^{-1} i H=2 D^{-1} H^{0,1} j(D),
$$

où la partie $(0,1)$ de l'opérateur $H$ est entendue en fonction des structures complexes $j(D)$ et $i$. Cette différentielle est surjective, car

$$
T_{j(D)} \mathcal{J}_{0}=\{K, K j(D)+j(D) K=0\},
$$

et $K=d_{D} j(H)$ équivaut à $H^{0,1}=-\frac{1}{2} D K j(D)$, équation qui possède une solution car l'opérateur $D K j(D)$ est $(0,1)$. De plus, il existe des constantes $\epsilon>0$ et $c$ indépendantes de $k$, telles que

$$
\nu\left(d_{D(s)} j\right) \geq \epsilon \text { et }|j|_{C^{2}} \leq c .
$$

Enfin, on a vu précédemment que $|j(D(s))-j| \leq C / \sqrt{k}$. En conclusion, le théorème des fonctions implicites nous donne l'existence d'un opérateur $\tilde{D}$ tel que

$$
j(\tilde{D})=j \text { et }|\tilde{D}-D(s)| \leq C / \sqrt{k} .
$$

Dans notre cas, au lieu de considérer les sections du fibré $G l\left(N_{0}, L^{k} \oplus L^{k}\right)$, on choisit les sections $d c$-équivariantes de ce fibré, c'est-à-dire les opérateurs $D$ tels que

$$
D_{c(x)} d_{x} c=d_{c(x)} D_{x}
$$

L'application $j$ étant équivariante, le résultat est qu'il est possible de trouver un $D$ équivariant résolvant $j(D)=j$. Maintenant, il suffit de perturber $s=s_{0} \oplus s_{1}$ en $\tilde{s}$ de façon équivariante sur un voisinage de $N$ de taille constante (pour la métrique $g_{k}$ ) afin que le nouveau $D(\tilde{s})$ soit égal à $\tilde{D}$. Ceci peut se faire sans changer l'ensemble $N$.

3.1.2. Propriété $(v)$. Nous suivons maintenant les pages 209 à 213 de [2], dont le contenu permet de perturber la fonction $F=s_{1} / s_{0}$ de sorte qu'elle convienne au modèle $(v)$ de la Définition 1.4. Pour cela, rappelons le contenu de la Proposition 9 de [2]:

Proposition 3.4. Soit $\Delta=\{\partial F=0\}, \Gamma=\{|\partial F| \leq|\bar{\partial} F|\}$, et enfin $\Omega_{\chi}=$ $\left\{\left|s_{0}\right| \geq \chi\right\}$. Alors

(1) $\Delta$ est un ensemble fini.

(2) Il existe $\chi$ et $\rho_{0}$ indépendants de $k$ tel que les $g_{k}$-boules centrées sur les points de $\Delta$ et de taille $\rho_{0}$ soient disjointes et contenues dans $\Omega_{\chi}$.

Fixons $x$ un point de $\Delta$. La dérivée covariante (induite par la connexion de Levi-Civita) de la 1-forme $\partial F$ se décompose de la façon suivante:

$$
\nabla(\partial F)=\partial_{\nabla}(\partial F)+\bar{\partial}_{\nabla}(\partial F) .
$$

Puisque l'involution est anti-holomorphe et isométrique, et que $F(c)=\bar{F}$, on obtient facilement que

$$
\overline{c^{*} \partial_{\nabla}(\partial F)}=\partial_{\nabla}(\partial F)
$$


Soit $H(z)=\sum H_{\alpha \beta} z_{\alpha} z_{\beta}$ un polynôme complexe en des coordonnés adaptées au point $x$ à $\omega$, et tel que sa hessienne en $x$ soit égale à $\partial_{\nabla}(\partial F)$. La perturbation de $F$ dans le cas classique sur $B_{g_{k}}(x, \rho)$ se fait de la façon suivante:

$$
\tilde{F}(z)=\beta_{\rho}(w+H(z))+\left(1-\beta_{\rho}\right) F(z),
$$

où $\beta_{\rho}$ est une fonction plateau à support dans $B_{g_{k}}(x, \rho)$ Le lemme de [2] décrit l'effet de cette modification:

Lemme 3.5. Pour $\rho$ assez petit, $k=k(\rho)$ assez grand, et $w$ assez proche (relativement à $\rho$ ) de $F(x)$, alors $x$ est le seul point de $B_{g_{k}}(x, \rho)$ où $|\partial F| \leq$ $|\bar{\partial} F|$.

Si $x$ appartient à $\Delta \cap \mathbb{R} X, F(x)$ est réel, si bien qu'il suffit de choisir $w$ réel pour que la perturbation précédente réalise la condition $(v)$, tout en laissant $F$ symétrique. Si $x$ appartient à $\Delta$ mais pas à $\mathbb{R} X$, on transforme $F$ en

$$
\tilde{F}(z)=\beta_{\rho}(w+H(z))+\left(1-\beta_{\rho}\right) F(z)+c^{*}\left(\beta_{\rho}(w+H(z))+\left(1-\beta_{\rho}\right) F(z)\right) .
$$

La Proposition 3.3 montre que si $x$ et $c(x)$ sont différents, alors ils sont forcément $g_{k}$-distants d'au moins $\rho_{0}$. Le lemme précédent montre alors que si $\rho$ est assez petit, les supports des deux parties de la fonction perturbatrice ne se rencontrent pas, si bien qu'on obtient sans effort la propriété $(v)$ en $x$ et $c(x)$. Par ailleurs en choisissant $w$ de sorte que $F(x)+w$ ne soit pas réel, on a $\tilde{F}(x) \neq \tilde{F}(c(x))$.

3.2. Démonstration de la Proposition 3.1. Les propriétés 1 et 2. La propriété 1 a été réalisée dans la première partie. Pour la propriété 2., nous reprenons les arguments de la partie 3.3 dans [5], (cf. aussi [6], ainsi que notre Proposition 2 avec $E=\mathbb{C}^{2}$ ). Le lieu des zéros $Z_{0, k}$ de $s_{0}$ est une hypersurface symplectique réelle, telle que la distance entre $T Z$ et $J T Z$ est en $1 / \sqrt{k}$. On peut alors appliquer la première partie à la suite de sections $s_{1 \mid Z_{0, k}}$, c'est-à-dire la rendre transverse sur $Z_{0, k}$ tout en la laissant symétrique pour la structure réelle induite par $c$. La morphologie de $Z_{0, k}$ varie avec $k$, mais cela ne pose pas de problèmes (cf. [5] pour les détails). Maintenant, si la restriction de $s_{1}$ est transverse, $s_{1}$ est également transverse dans l'espace ambiant aux points de $Z_{0, k}$. Enfin, on peut constater qu'alors la section perturbée $s_{0} \oplus s_{1}$ est transverse sur un voisinage fixe de $Z_{0, k}$.

Propriété 3: la transversalisation de $\partial\left(s_{1} / s_{0}\right)$. Il reste à transversaliser la section $f=\partial\left(s_{1} / s_{0}\right)$. Cela se fait sur trois types d'endroits : hors d'un voisinage de $g$-taille fixe, sur un $g_{k}$-voisinage fixe de $\mathbb{R} X$, et enfin entre les deux précédents. Dans le premier cas, nous suivons la construction de Donaldson. Etant donné que d'une part les supports des sections $s_{k}$ et $\kappa\left(s_{k}\right)$ sont disjoints, et que d'autre part la condition de transversalité de $\partial\left(s_{1} / s_{0}\right)$ est invariante par $c$, il suffit de réaliser la perturbation sur la moitié 
des boules, et de perturber par $g+\overline{c^{*} g}$ plutôt que simplement par $g$. La transversalisation dans la dernière situation intermédiaire est réalisée dans [6, p. 22]. Leur travail s'adapte immédiatement à notre cas. En ce qui concerne la seconde situation, dans le cas traité par [6], la paire de sections est telle que la restriction de leur rapport est une fonction de Morse donnée sur $\mathbb{R} X$, si bien que la transversalisation de $f$ est déjà faite. Dans notre cas, nous partons de deux sections quelconques, si bien qu'il est nécessaire de transversaliser près de $\mathbb{R} X$. Néanmoins, nous utilisons essentiellement la méthode des trois auteurs précédemment cités. La transversalisation de $f$ par [6] utilise une récurrence de la façon suivante. Soit $U$ un ouvert connexe, simplement connexe et invariant par $c$. Choisissons des sections $\alpha_{i}$ orthonormales du fibré trivial $T^{1,0} X_{\mid U}$, et nommons $A_{r}$ le fibré en droites complexes engendrées par les valeurs de $\alpha_{r}$. On a donc $\bigoplus_{r=1}^{r=n} A_{r}=T^{1,0} X_{\mid U}$. Soit de plus $\pi_{\leq r}$ la projection de $T^{1,0} X$ sur $E_{r}=\bigoplus_{i \leq r} A_{r}$, et $\pi_{r}$ la projection sur $A_{r}$.

Le principe de récurrence utilise le lemme suivant:

Lemme 3.6. [5]. Si s est une section de $E \otimes L^{k}$ transverse sur $W=\{s=0\}$, et $\tau$ une section $A H$ de $L^{k}$ telle que $t_{\mid W}$ est transverse sur $W$, alors $s \oplus t$ est transverse.

Ainsi, on suppose que $\pi_{\leq r} f$ est transverse sur $W_{r}=\left\{\pi_{\leq r} f=0\right\}$. On tente ensuite de perturber $F$ pour obtenir la transversalisation sur $W_{r}$ de $\pi_{r+1} f$. Au bout de $n$ itérations, on obtient la transversalisation de $F$ sur l'ouvert. Supposons donc que $\pi_{\leq r} f$ soit transverse sur $W_{r}$. Nous recouvrons l'intersection de $W_{r}$ avec le voisinage de $\mathbb{R} X$ par des boules centrées sur des points de $\mathbb{R} X$ (et non pas sur des points de $W_{r}$ comme dans $[\mathbf{6}]$ ).

Soit $x \in \mathbb{R} X$ un de ces centres, et $\left(z_{i}\right)$ des coordonnées complexes centrées $x$, telles que pour tout $i, \partial z_{i}(x)=\alpha_{i}(x)$. Puis perturbons $s_{1}$ de la façon suivante:

$$
s_{1}^{\prime}=s_{1}+w z_{r+1} \sigma_{k, x} .
$$

On a alors $\partial\left(s_{1}^{\prime} / s_{0}\right)=\partial\left(s_{1} / s_{0}\right)+w\left(\sigma_{k, x} / s_{0} \partial\left(z_{r+1}\right)+z_{r+1} \partial\left(\sigma_{k, x} / s_{0}\right)\right)$. Rappelons qu'il est nécessaire de transversaliser $f$ uniquement sur $X_{\epsilon, k}=\left\{\left|s_{0}\right| \geq\right.$ $\epsilon\}$. Si on appelle $\zeta$ la section de $A_{r+1}$ définie par

$$
\zeta=\left(\sigma_{k, x} / s_{0}\right) \pi_{r+1}\left(\partial z_{r+1}\right)+z_{r+1} \pi_{r+1}\left(\partial\left(\sigma_{k, x} / s_{0}\right)\right),
$$

alors $\zeta$ est uniformément minorée sur $W_{r} \cap X_{\epsilon, k} \cap B_{g_{k}}(x, \rho)$, si $\rho$ est suffisamment petit (par rapport à $\epsilon$ et indépendamment de $k$ et $x$ ). En effet, l'estimation $\left|\partial\left(\sigma_{k, x} / s_{0}\right)\right| \leq C \sqrt{k}$ ( $C$ dépend cette fois de $\epsilon$ ) montre que pour $\rho$ assez petit (par rapport à $\epsilon$ ), la seconde moitié de $\zeta$ est inférieure en norme à $1 / 10$ de la norme de la première partie, qui est minorée par une constante strictement positive dépendant de $\epsilon$. La section

$$
\pi_{r+1}\left(\partial\left(s_{1}^{\prime} / s_{0}\right)\right)=\pi_{r+1}\left(\partial\left(s_{1} / s_{0}\right)\right)+w \zeta
$$


est donc bien définie sur l'intersection de $W_{r}$ avec la boule $B_{g_{k}}(x, \rho)$ et $X_{\epsilon, k}$, ainsi que la fonction

$$
f_{w}=\frac{\pi_{r+1} \partial\left(s_{1} / s_{0}\right)}{\zeta}+w
$$

Pour toute composante connexe $C$ de l'intersection de $W_{r, w}$ avec la boule $B_{k}(x)$, on choisit une trivialisation $(\mathrm{cf} .[\mathbf{5}, \mathbf{6}])$ de $C$. La restriction à celle-ci de la fonction possède les estimées d'holomorphie asymptotique. Maintenant la Proposition 2.13 permet de trouver un $w$ réel tel que $f_{w \mid C}$ devienne $\eta$ transverse sur $C$. On recommence le processus pour toutes les composantes, en nombre borné indépendant de $k$ (mais dépendant de $\epsilon$ ), pour transversaliser $f_{w \mid W_{r, w}}$. Au total, la section

$$
\pi_{r+1}\left(\partial\left(s_{1} / s_{0}\right)+w \pi_{r+1} \partial\left(z_{r+1} \sigma_{k, x} / s_{0}\right)\right.
$$

est symétrique sur $X$, et transverse sur $W_{r, w}$. Par récurrence, on obtient donc le résultat.

\section{References}

[1] S.K. Donaldson, Symplectic submanifolds and almost-complex geometry, J. Diff. Geom. 44 (1996), 666-705.

$[2]-$ Lefschetz pencils on symplectic manifolds, J. Diff. Geom. 53 (1999), 205-236.

[3] J-Y. Welschinger, Invariants of real symplectic 4-manifolds and lower bounds in real enumerative geometry, Inv. Math. 162, (2005), 195-234.

[4] - Real structures on minimal ruled surfaces, Comment. Math. Helv. 78 (2) (2003), 418-446.

[5] D. Auroux, Asymptotically holomorphic families of symplectic submanifolds, GAFA 7 (1997), 971-995.

[6] D. Auroux, V. Muñoz and F. Prezas, Lagrangian submanifolds and Lefschetz pencils, arXiv:math.SG/0407126.

[7] D. Auroux, D. Gayet and J.-P Mohsen, Symplectic hypersurfaces in the complement of an isotropic submanifold, Math. Ann. 321 (2001), 739-754.

[8] D.A. Edelman and E. Kostlana, How many zeros of a random polynomial are real ?, Bull. AMS 32, (1995), 1-37.

UNIVERSITÉ DE LYON

UNIVERSITÉ LYON 1

Institut CAmille JoRdan CNRS UMR 5208

43 BOULEVARD DU 11 NOVEMBRE 1918

69622 Villeurbanne CEDEX France

E-mail address: gayet@math.univ-lyon1.fr

Received 5/21/2007, accepted 3/11/2008 